\title{
Propriedades psicométricas da Philadelphia Mindfulness Scale considerando fumadores e não fumadores portugueses
}

\section{Psychometric properties of the Philadelphia Mindfulness Scale considering Portuguese smokers and non-smokers}

H. Rodrigues-Fernandes, H. Pereira, L. Almeida, C. Teixeira, J. Vasconcelos-Raposo

\author{
ARTIGO ORIGINAL | ORIGINAL TITLE
}

\begin{abstract}
RESUMO
O mindfulness, enquanto processo psicológico, tem sido alvo de um interesse crescente por parte da investigação científica. O objetivo do presente estudo foi o de examinar a confiabilidade, validade fatorial e medição da invariância (fumadores e não fumadores) de uma versão portuguesa da Philadelphia Mindfulness Scale (PHLMS). Na pesquisa participaram 315 adultos portugueses entre os 18 e os 62 anos $(\mathrm{M}=23.14$; $\mathrm{DP}=7.286)$, sendo 110 fumadores e 205 não fumadores. A confiabilidade para a dimensão consciência foi de .861 e de .868 para a subescala aceitação. A análise fatorial confirmatória apresentou um modelo ajustado a dois fatores sem necessidade de eliminação de itens. A análise multigrupos suportou a invariância do instrumento entre fumadores e não fumadores. Os resultados evidenciam boas propriedades psicométricas para a utilização da PHLMS no contexto de investigação em amostras de adultos portugueses.
\end{abstract}

Palavras-chave: Philadelphia Mindfulness Scale, análise fatorial confirmatória, invariância, fumadores portugueses

\begin{abstract}
Mindfulness, as a psychological process, has been a subject of a growing interest in scientific research. This study aimed to examine the reliability, factor validity, and measurement invariance (smokers and non-smokers) a Portuguese version of Philadelphia Mindfulness Scale (PHLMS). The sample consisted of 315 Portuguese adults between 18 and 62 years $(M=23.14$; $S D=7.286)$ whit 110 smokers and 205 non-smokers. The estimate of reliability for awareness size was .861 and .868 for the subscale acceptance. The confirmatory factor analysis showed a model adjusted to two factors without removing items. The multi-groups analysis supported the invariance of the instrument between smokers and non-smokers. The results show significant psychometric properties of PHLMS for use in research in Portuguese samples of adults.

Keywords: physical activity, physical exercise, self-concept, school performance
\end{abstract}

Hugo Rodrigues-Fernandes. Health \& Performance Psych Lab, Universidade de Trás-os-Montes e Alto Douro, Vila Real, Portugal. e-mail: hugorodriguesfernandes.psi@outlook.com

Helena P. Pereira. Faculdade de Medicina da Universidade do Porto, Porto, Portugal.

Luís Almeida. Faculdade de Desporto, Faculdade de Psicologia e de Ciências da Educação, Universidade do Porto, Porto, Portugal.

Carla M. Teixeira, José Vasconcelos-Raposo. ECHS, INESC-TEC MASSIVE Lab. Universidade de Trás-os-Montes e Alto Douro, Vila Real, Portugal. 
Disseminado a partir da tradição cultural budista e constituindo-se como um fator mental, passível de se apresentar em dado instante na consciência (Kabat-Zinn, 2003; Rosch, 2007; Silveira et al., 2012), os processos associados ao mindfulness têm sido alvo de um crescente interesse por parte da investigação científica (Kabat-Zinn, 2003; Williams \& Kabat-Zinn, 2011; Williams \& Penman, 2011). Desde os estudos iniciais e com a sua introdução nas psicoterapias da terceira geração (Kabat-Zinn, 2003), o mindfulness é visto como um conjunto de processos metacognitivos capaz de influenciar inúmeros processos mentais (Brewer et al., 2013; Williams \& Kabat-Zinn, 2011). Como intervenção complementar tem apresentado resultados consistentes ao nível da redução de sintomatologia associada à ansiedade e depressão (Kabat-Zinn, 2003; Segal et al., 2012), de problemas alimentares (Butryn et al., 2013) e no tratamento de pacientes aditivos (Brewer et al., 2013; Elwafi et al., 2012; Luberto et al., 2014).

O Mindfulness difere da noção de esquemas, narrativas ou crenças, pois entende-se que estes termos englobam a utilização de um pensamento crítico e reflexivo (Silveira et al., 2012). Essencialmente, o mindfulness refere-se à capacidade do indivíduo em orientar a consciência segundo um foco de atenção para o momento presente, com aceitação e sem julgamento (Bishop et al., 2004; Kabat-Zinn, 2003; Williams \& Penman, 2011). Estas atitudes são entendidas como cruciais para a mudança de comportamentos e para a promoção do bem-estar (Cardaciotto et al., 2008).

Dado o crescente interesse terapêutico e científico em torno das técnicas baseadas no treino em mindfulness, vários instrumentos de autorrelato têm sido produzidos para mensurar o construto nas suas várias dimensões, entretanto, propostas (Baer et al., 2008; Lau et al., 2006). Importa salientar que algumas das escalas desenvolvidas e aferidas surgiram no sentido de avaliar o mindfulness em indivíduos praticantes de técnicas terapêuticas de meditação baseadas no mindfulness (Lau et al., 2006; Walach et al., 2006). Posto isto, a pertinência do desenvolvimento da Philadelphia Mindfulness Scale (PHLMS) deveu-se à necessidade de propor um instrumento que incidisse sobre a avaliação de pessoas não praticantes de técnicas associadas ao treino de mindfulness e ao mesmo tempo auxiliar a investigação científica (Cardaciotto et al., 2008; Teixeira \& Pereira, 2012; Teixeira et al., 2017). Fundamentalmente, a PHLMS surgiu da crença sobre a possibilidade de medir o mindfulness enquanto processo natural da pessoa (Bishop et al., 2004; Cardaciotto et al., 2008).

São escassos os estudos que avaliaram psicometricamente a PHLMS, embora já tenha sido adaptada para a população brasileira (Silveira et al., 2012), portuguesa (Teixeira et al., 2017) e espanhola (Tejedor et al., 2014), tendo sido efetuados trabalhos sobre a escala com a população budista chinesa (Zeng et al., 2015). Quanto a análises comparativas, Cardaciotto et al. (2008) consideraram populações clínicas, tal como Tejedor et al. (2014) que integrou comparações ao nível de perturbações psiquiátricas e mais recentemente os trabalhos de Morgan et al. (2020) que avaliaram o comportamento da escala entre praticantes e não praticantes de meditação. Destes, para além do estudo inicial (Cardaciotto et al., 2008) nenhuma investigação apresentou resultados referentes à Análise Fatorial Confirmatória (AFC) da escala. Neste contexto, como alerta a literatura (Fernandes \& Vasconcelos-Raposo, 2010; Marôco, 2021; Teixeira et al., 2020; Vasconcelos-Raposo et al., 2013; Vasconcelos-Raposo et al., 2012) este procedimento é essencial para avaliar de um modo robusto a estrutura fatorial de instrumentos em investigação. Já Silveira et al. (2012) alertaram para a necessidade de sistematizar estudos psicométricos atendendo a caraterísticas específicas das amostras 
para aprimorar o conhecimento em torno do comportamento da PHLMS.

Morgan et al. (2020) reportaram mais de 53 estudos empíricos que já recorreram à PHMLS, desde a sua conceção (Cardaciotto et al., 2008). Num deles, Bloise et al. (2016) aplicaram dois programas baseados em mindfulness identificando potencialidades no desenvolvimento de processos mentais associados à consciência e à aceitação, através de movimentos educativos baseados na prática de yoga. Mais recentemente, Tronieri, et al. (2020) verificaram a aceitação como um preditor determinante para a perda de peso de curto-prazo, num estudo longitudinal que acompanhou 178 adultos, num programa de redução de peso e promoção de estilos de vida. Estes e outros estudos têm assim permitido perceber uma série de fenómenos relacionados com a saúde e o mindfulness (Morgan et al., 2020).

$\mathrm{Na}$ composição conceptual do mindfulness, existe um amplo debate quanto às dimensões do construto (Cardaciotto et al., 2008; Tejedor et al., 2014). Por exemplo, para Baer et al. (2008) a mensuração do mindfulness é efetuada considerando cinco fatores (observar, descrever, agir com consciência, não reagir face à experiência do aqui e do agora). Já a Mindfulness Attention and Consciência Scale (Brown \& Ryan, 2003) apesar de apresentar bons índices psicométricos, recebeu algumas críticas por não integrar componentes de mensuração do mindfulness, nomeadamente no que se refere à aceitação (Silveira et al., 2012; Walach et al., 2006). Lau et al. (2006) apresentaram uma escala de medida de mindfulness válida para medir o construto em praticantes de treino em mindfulness, limitando a análise em populações sem experiência em meditação (Cardaciotto et al., 2008). Já Walach et al. (2006) apresentaram um instrumento que mede o mindfulness num único fator, sugerindo uma forma longa para sujeitos treinados em mindfulness e outra abreviada para investigação. Esta sugestão unifatorial criticada, já que torna a operacionalização do conceito ambígua, quando se pretende compreendê-lo de um modo holístico (Silveira et al., 2012).

Alguns trabalhos sugerem que o desenvolvimento dos processos mentais inerentes ao mindfulness é possível graças a práticas terapêuticas que recorrem à meditação (Grossman \& Van Dam, 2011), ao passo que outras investigações frisam uma vertente inata e natural do desenvolvimento do construto, não descurando o fator aprendizagem em contexto terapêutico (Brown \& Ryan, 2003; Cardaciotto et al., 2008). Aliás, outro dos propósitos da elaboração da PHLMS foi o de dar resposta a uma carência da época, em termos de investigação, que era o de propor instrumentos passíveis de medir o mindfulness em pessoas sem grandes conhecimentos resultantes do envolvimento em meditação baseada em mindfulness (Cardaciotto et al., 2008).

Em análise aos estudos prévios de validação da PHLMS (Cardaciotto et al., 2008; Silveira et al., 2012; Teixeira et al., 2017; Tejedor et al., 2014; Zeng et al., 2015), todos eles têm sido unânimes quanto à atribuição de uma organização bifatorial da escala. Em termos de consistência interna e externa os valores têm sido satisfatórios, definindo a PHLMS como um inventário simples, curto e objetivo na mensuração do mindfulness, útil para a pesquisa científica (Silveira et al., 2012; Tejedor et al., 2014).

No intuito de aumentar o conhecimento em torno das caraterísticas psicométricas da PHLMS, a sistematização de estudos em amostras específicas mostra-se pertinente e útil (Silveira et al., 2012). Neste contexto, sendo o consumo de tabaco um dos fatores de risco para várias doenças (Buffart et al., 2014), tem sido crescente o esforço em adaptar a meditação baseada em mindfulness como uma técnica terapêutica promotora da cessação tabágica (Elwafi et al., 2012; Luberto et al., 2014). Por exemplo, para Luberto et al., (2014) trabalhar a capacidade de aceitação e de abertura à experiência, mediante uma atitude de foco no aqui e agora, revela-se útil no desenvolvimento de competências para lidar com 
eventos emocionais negativos e assim manter a abstinência. Segundo Elwafi et al. (2012) existe uma forte correlação entre a prática de mindfulness e a diminuição do desejo de fumar. Embora os estudos demonstrem potencial sobre a aplicação de técnicas de mindfulness em indivíduos motivados para a cessação tabágica (Elwafi et al., 2012; Luberto et al., 2014) pouco se conhece de evidências psicométricas dos instrumentos em fumadores.

Da revisão da literatura levada a cabo realça-se a importância em se aumentar o conhecimento em torno das caraterísticas psicométricas da PHLMS a partir do teste ao modelo teórico proposto por Cardaciotto et al. (2008), com recurso a uma AFC. Para além disso, apresentar análises de invariância na comparação entre fumadores e não fumadores revela-se importante, dado o potencial da intervenção baseada em mindfulness nos programas de cessação tabágica (Elwafi et al., 2012; Luberto et al., 2014) e a carência de estudos sobre as caraterísticas psicométricas da PHLMS naquela população.

O estudo tem por objetivo geral apresentar as propriedades psicométricas da PHLMS (Cardaciotto et al., 2008) numa amostra de adultos portugueses, considerando sujeitos fumadores e não fumadores. Especificamente, pretende-se confirmar a estrutura fatorial da PHLMS, verificando as propriedades psicométricas do instrumento (AFC, evidências de confiabilidade e de validade bifatorial, validade convergente e discriminante). Pretende-se ainda verificar a invariância fatorial do instrumento em pessoas fumadoras e não fumadoras.

\section{MÉTODOS}

Este estudo baseia-se no paradigma correlacional, uma vez que é pretendido compreender o grau e a forma de associação entre as variáveis analisadas (Marôco, 2021; Pais-Ribeiro, 2010). É, ainda, um estudo do tipo transversal e comparativo, dado as variáveis terem sido medidas num único espaço temporal (Pais-
-Ribeiro, 2010) e por procurarmos verificar hipotéticas diferenças no comportamento do instrumento entre grupos de fumadores e não fumadores que constituem a presente amostra.

\section{Amostra}

No que se refere à caraterização da amostra (Quadro 1), esta foi constituída por um total de 315 adultos portugueses $(66.3 \%$ do sexo feminino) com idades entre os 18 e os 62 anos $(\mathrm{M}=23.14 ; \mathrm{DP}=7.286)$, provenientes de uma instituição de ensino superior pública, da região norte portuguesa. Dos participantes, $47.0 \%$ residiam em zonas rurais e os restantes $53 \%$ em zonas urbanas. Considerando as habilitações literárias completas dos indivíduos, $2.5 \%$ dos indivíduos tinham habilitações até ao terceiro ciclo, $67.3 \%$ o ensino secundário e os restantes $30.2 \%$ haviam completado uma formação superior. Do total de participantes, 110 pessoas eram fumadoras (56.9 \% do sexo feminino) com média de idades de 26.28 anos. Quanto à dependência de nicotina, $59.6 \%$ apresentaram baixos níveis de dependência e $40.4 \%$ indicadores para dependência média-alta.

Quadro 1

Caracterização da amostra.

\begin{tabular}{lcc}
\hline & $\mathrm{N}$ & $\%$ \\
& 315 & 100 \\
\hline Sexo & & \\
$\quad$ Feminino & 209 & 66.3 \\
$\quad$ Masculino & 106 & 33.7 \\
Local de residência & & \\
$\quad$ Rural & 148 & 47.0 \\
$\quad$ Urbano & 167 & 53.0 \\
Habilitações literárias (completas) & & \\
$\quad$ Até ao ensino secundário & 220 & 69.8 \\
$\quad$ Ensino superior & 95 & 30.2 \\
Fumadores & & \\
$\quad$ Não & 205 & 65.1 \\
$\quad$ Sim & 110 & 34.9 \\
Nível de dependência & & \\
$\quad$ Dependência baixa & 65 & 59.6 \\
$\quad$ Dependência média-alta & 45 & 40.4 \\
\hline
\end{tabular}




\section{Instrumentos}

Para cumprir os propósitos do estudo recorremos a um protocolo de autorrelato composto por um questionário sociodemográfico, bem como um instrumento para medir o nível de dependência tabágica e diferenciar pessoas fumadoras de não fumadoras (Fagerström, 1978), assim como a PHLMS (Cardaciotto et al., 2008).

\section{Dados sociodemográficos}

Para o levantamento das variáveis independentes e de dados sociodemográficos desenvolveu-se um questionário que incluiu questões relativamente ao sexo, idade, zona de residência, local de residência, habilitações literárias e consumo atual de tabaco.

\section{Dependência Tabágica}

Para conhecer os níveis de dependência tabágica dos fumadores recorreu-se ao Teste de Dependência de Nicotina (Fagerström, 1978). Este consiste num instrumento de autorrelato, composto por seis itens, fácil de aplicar e útil para conhecer o nível de dependência do indivíduo à nicotina (Rocha et al., 2010). Segundo Rocha et al. (2010) a pontuação total da escala é de 10 pontos, sugerindo uma classificação dos indivíduos fumadores em três categorias: 1- baixa dependência fisiológica (0-2 pontos); 2- dependência moderada (3-6 pontos); 3dependência elevada (7-10 pontos).

\section{Mindfulness (PHLMS)}

A PHLMS (Cardaciotto et al., 2008) é uma medida de autorrelato de 20 itens que avalia, segundo um modelo bifatorial, dimensões inerentes ao mindfulness (Cardaciotto et al., 2008), nomeadamente consciência (consciência do momento presente) e aceitação. Os itens são agregados a uma tipologia de escala do tipo Likert (0- nunca a 4- quase sempre), que mede a frequência de vivências tendo por referência a última semana. É ainda sugerida a inversão dos valores de todos os itens pares (Cardaciotto et al., 2008). Os estudos têm apontado bons índices de confiabilidade da escala (Cardaciotto et al., 2008; Tejedor et al., 2014, Teixeira et al., 2017). Por exemplo, Cardaciotto et al. (2008) apresentaram valores de alfa de Cronbach de .75 para a subescala consciência e .82 para a aceitação.

\section{Procedimentos}

O projeto de pesquisa obteve parecer favorável da Comissão de Ética Institucional de Pesquisa da Universidade de Trás-os-Montes e Alto Douro. Os participantes foram convidados a responder ao protocolo de investigação, tendo sido informados relativamente aos propósitos da pesquisa, através de leitura e resposta ao termo de consentimento livre e esclarecido, onde o anonimato e a confidencialidade foram garantidos, bem como o direito de desistir ou de solicitar o apoio do investigador, no decorrer da recolha de dados. Foi também garantida a não existência de riscos ou danos para os participantes, sendo a sua participação voluntária e sem qualquer compensação ou contrapartida direta. O momento de recolha de dados demorou cerca de 15 minutos.

\section{Análise Estatística}

Para o tratamento estatístico dos dados foram utilizados os softwares SPSS (v. 27) e o AMOS (v. 27, SPSS, IBM Company, Chicago, IL). De início, efetuou-se a análise às estatísticas descritivas referentes aos itens das escalas, assim como às variáveis em análise, a partir da média $(M)$ e do desvio padrão $(D P)$. A análise da simetria da distribuição das frequências (normalidade univariada) foi realizada através dos coeficientes skewness (assimetria) e kurtosis (achatamento). Calculou-se o grau de consistência interna das escalas, recorrendo ao alfa de Cronbach e à Fiabilidade Compósita (FC).

Tendo por referência as estruturas fatoriais propostas nos estudos prévios (Cardaciotto et al., 2008), realizou-se a AFC. Foi ainda realizada uma AFC de segunda ordem, para verificar a hipótese de estabelecimento de um 
modelo hierárquico para a versão portuguesa da PHLMS. No que se refere aos índices de modificação foram atendidos a correlação de itens com valores superiores a dez (Marôco, 2021). Para os modelos testados foi utilizado o método de estimação maximum likelihood. Já no sentido de verificar a adequabilidade do modelo aos dados, seguindo as recomendações de Byrne (2001) e Marôco (2021) utilizaram-se as seguintes medidas de avaliação do ajustamento: ratio chi-square statistics/degrees of freedom $\left(X^{2} / d f\right)$, comparative fit index (CFI), goodness of fit index (GFI), root mean square error of approximation (RMSEA), o maximum expected cross-validation index (MECVI) e o akaike information criterion (AIC). $\mathrm{O}$ rácio do $X^{2} / d f$ tem sido usado como índice de ajustamento do modelo, não existindo, porém, consenso acerca do valor que se considera um ajustamento adequado, sendo sugerido valores inferiores a 3 (Aroian \& Norris, 2005; Marôco, 2021). O CFI avalia a adequabilidade do modelo em relação ao modelo independente, variando os valores entre 0 e 1 , com valores superiores a .90 a propor um ajustamento adequado. Já o GFI mede a quantidade relativa de variância e covariância conjuntamente explicadas pelo modelo, varia entre 0 e 1 , com valores superiores a .90 a sugerir um ajustamento adequado. Quanto ao RMSEA, analisa a discrepância no ajustamento entre as matrizes estimadas e as observadas, varia entre 0 e 1 , sendo que os valores esperados devem situar-se abaixo de .08. O MECVI corresponde ao índice de amostra única de validação cruzada de Browne e Cudeck (1989). Neste caso, quanto menor o valor apresentado, melhor o ajustamento do modelo (Byrne, 2001). O AIC indica o grau de parcimónia ou simplicidade do modelo testado e tal como acontece com o MECVI, quanto menor for o valor obtido melhor é o modelo.

Para a invariância fatorial da PHLMS, inicialmente definimos um modelo de adequação apropriado para ambos os grupos comparativos (fumadores vs. não fumadores). Após a obtenção dos fatores, estes foram submetidos simultaneamente a uma análise multigrupos (com correção Emulisrel6) no sentido de efetuar um conjunto progressivo de restrições (cargas fatoriais, variâncias e covariâncias), de forma a analisarmos a equivalência do instrumento para os diferentes subgrupos (fumadores vs. não fumadores). Os valores da diferença do Qui-quadrado $(\Delta \chi 2)$ e respetivos graus de liberdade ( $d f$ ) foram utilizados para analisar possíveis diferenças significativas entre os diferentes modelos formulados. Para uma observação mais detalhada dos procedimentos de análise multigrupos, pode-se consultar outras referências tidas em consideração (Byrne, 2001; Fernandes \& Vasconcelos-Raposo, 2010; Marôco, 2021; Vasconcelos-Raposo et al., 2012).

\section{RESULTADOS}

A Quadro 2 apresenta a análise descritiva inicial realizada em todos os itens do questionário para a amostra total $(n=315)$.

Quadro 2

Análise descritiva dos itens da PHLMS-R.

\begin{tabular}{lccc}
\hline Item & $\mathrm{M} \pm \mathrm{DP}$ & Skewness & Kurtosis \\
\hline 1_cons & $3.25 \pm .801$ & -.864 & .336 \\
2_ace & $1.15 \pm .961$ & .607 & .038 \\
3_cons & $3.04 \pm .891$ & -.685 & .234 \\
4_ace & $2.35 \pm 1.131$ & -.367 & -.512 \\
5_cons & $2.76 \pm 1.280$ & -.690 & -.702 \\
6_ace & $2.06 \pm 1.163$ & -.124 & -.797 \\
7_cons & $2.18 \pm 1.663$ & -.088 & -.731 \\
8_ace & $1.83 \pm 1.58$ & .286 & -.811 \\
9_cons & $2.72 \pm 1.128$ & -.497 & -.620 \\
10_ace & $1.92 \pm 1.150$ & .029 & -.714 \\
11_cons & $2.56 \pm .990$ & -.262 & -.487 \\
12_ace & $1.83 \pm 1.065$ & .022 & -.732 \\
13_cons & $2.74 \pm .894$ & -.344 & -.096 \\
14_ace & $1.58 \pm 1.135$ & .271 & -.700 \\
15_cons & $2.66 \pm 1.098$ & -.399 & -.625 \\
16_ace & $1.61 \pm 1.124$ & .272 & -.642 \\
17_cons & $2.54 \pm .961$ & -.287 & -.083 \\
18_ace & $1.77 \pm 1.068$ & .110 & -.519 \\
19_cons & $2.78 \pm .906$ & -.432 & -.073 \\
20_ace & $1.38 \pm 1.050$ & .500 & -.306 \\
\hline Notat cons- cons & &
\end{tabular}

Nota: cons- consciência; ace- aceitação 
A estatística descritiva (mínimo, máximo, médias e desvios-padrão) e medidas de normalidade univariada (assimetria e achatamento) para a versão portuguesa da PHLMS, considerando amostra total, situaram-se nos $27.23 \pm 6.80$ na subescala consciência e nos $17.23 \pm 7.48$ para a subescala aceitação, enquanto a média da escala geral foi de 44.46 \pm 8.49. Quanto aos coeficientes de normalidade univariada variou entre -.135 e .355 no teste Skewness e entre -.142 e .246 no teste Kurtosis. A fim de examinar o grau de confiabilidade das escalas, computaram-se estimativas quanto ao alfa de Cronbach. Assim, os valores de alfa foram definidos a .86 para a subescala consciência, .87 para a aceitação e .72 para a PHLMS geral, considerando o total de casos da amostra em estudo.

Tendo por base os modelos sugeridos anteriormente pelas investigações que estudaram a PHLMS (Cardaciotto et al., 2009; Silveira et al., 2012; Tejedor et al., 2014), foram testados diversos modelos bifatoriais e um modelo hierárquico de segunda ordem, seguidamente reportados. Avaliou-se ainda a invariância entre grupos de fumadores e não fumadores (Quadro 3). O modelo 1, ajustado a uma amostra de 315 adultos portugueses, revelou uma qualidade de ajustamento sofrível $\left(\chi^{2} / \mathrm{df}\right.$ $=2.908$; $\mathrm{CFI}=.864 ; \mathrm{GFI}=.845$; $\mathrm{RMSEA}=$ .078 ; $\mathrm{MECVI}=1.845$; $\mathrm{AIC}=573.45)$. Atendendo aos índices de modificação e correlacionados os erros de medida (Modelo 2) atingiu-se uma boa qualidade de ajustamento $\left(\chi^{2} / \mathrm{df}\right.$ $=2.026$; $\mathrm{CFI}=.931 ; \mathrm{GFI}=.905$; $\mathrm{RMSEA}=$ .057; MECVI $=1.374$; $\mathrm{AIC}=423.80)$. Atendendo à análise e considerando a comparação por grupo de pessoas fumadoras com não fumadoras (Modelo 3) atingiu-se um ajustamento adequado do modelo $\left(\chi^{2} / \mathrm{df}=1.677\right.$; $\mathrm{CFI}=.912$; GFI $=.858 ;$ RMSEA $=.047$; MECVI $=2.476$; AIC $=738.07$ ). Para a análise da invariância considerando pessoas fumadoras e não fumadoras, o modelo constrito com pesos fatoriais, interceptos e variâncias/ covariâncias fixas entre as amostras não apresentou um ajustamento significativamente pior quando comparado ao modelo com parâmetros livres $\left(\Delta \chi^{2} \lambda(18)=21.338, p=.263\right.$; $\Delta \chi^{2} \mathrm{i}(38)=36.573, p=.535 ; \Delta \chi^{2} \operatorname{cov}(41)=$ 38.110, $p=.600$ ). Fica patente a invariância de medida forte do modelo bifatorial da versão portuguesa da PHLMS em pessoas fumadoras e não fumadoras.

Quadro 3

Índices de ajustamento das AFC aos diferentes modelos.

\begin{tabular}{|c|c|c|c|c|c|c|}
\hline Modelo Bi-fatorial & $\chi^{2} / \mathrm{df}$ & GFI & CFI & RMSEA & MECVI & AIC \\
\hline 1- Modelo sem correlação de erros & 2.908 & .864 & .845 & .078 & 1.845 & 573.45 \\
\hline 2- Modelo com correlação de erros & 2.026 & .931 & .905 & .057 & 1.374 & 423.80 \\
\hline 3- Amostra F vs. NF & 1.677 & .912 & .858 & .047 & 2.476 & 738.07 \\
\hline Invariância F vs. NF & \multicolumn{2}{|c|}{$\Delta \chi^{2}$} & \multicolumn{2}{|c|}{ Df } & \multicolumn{2}{|c|}{$p$} \\
\hline Pesos fatoriais & \multicolumn{2}{|c|}{21.338} & \multicolumn{2}{|c|}{18} & \multicolumn{2}{|c|}{.263} \\
\hline Interceptos de medida & \multicolumn{2}{|c|}{36.573} & \multicolumn{2}{|c|}{38} & \multicolumn{2}{|c|}{.535} \\
\hline Covariâncias estruturais & \multicolumn{2}{|c|}{38.110} & \multicolumn{2}{|c|}{41} & \multicolumn{2}{|c|}{.600} \\
\hline
\end{tabular}

Nota: $\chi 2=$ chi-square; $\chi^{2} / \mathrm{df}=\mathrm{CFI}=$ Comparative Fit Index; RMSEA = Root Mean Square Error of Approximation; MECVI - Maximum Expected Cross-Validation Index; AIC = Akaike information criterion; $\Delta \chi^{2}=$ different value of qui-square; F vs. NF = Fumadores vs. Não Fumadores.

Foi ainda calculada a FC, a validade convergente e a discriminante. Relativamente à FC o valor obtido foi .878 para a dimensão cons- ciência e .880 para a aceitação. O modelo oblíquo foi validado, não se provando a validade convergente, verificando-se a vali- 
dade discriminante da escala, dada a relação negativa (-.36) entre as variáveis latentes do modelo proposto. O modelo validado está representado na figura 1.

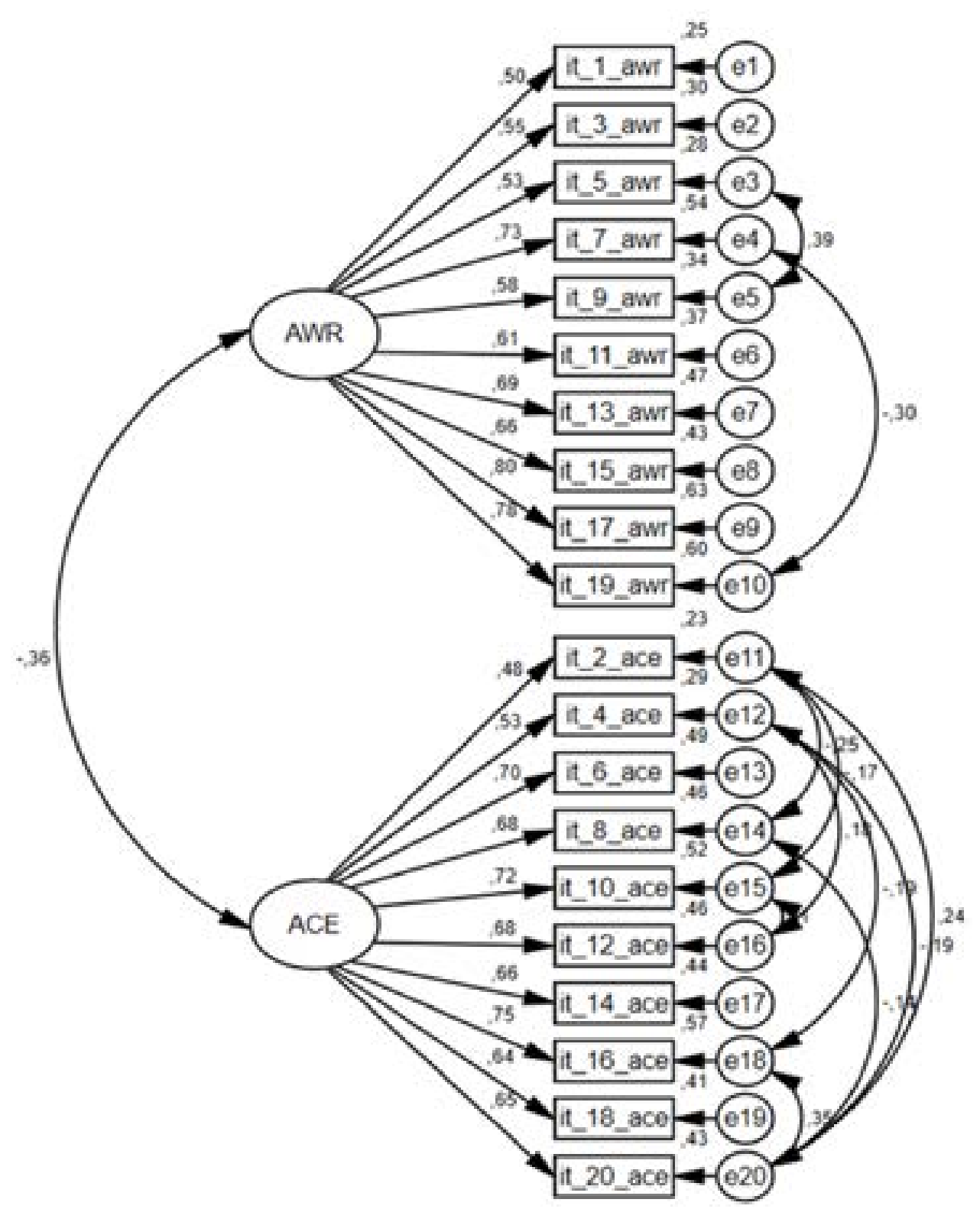

Figura 1. Modelo bifatorial da PHLMS para uma amostra portuguesa.

Quanto à validade discriminante, determinada pela relação entre os valores de VEM e do $r^{2}$ calculado entre as variáveis, verificou-se que os valores de $\mathrm{VEM}(\mathrm{AWR}=.423 ; \mathrm{ACE}=.427$ são superiores ao $r^{2}(.108)$ permitindo, assim, confirmar a existência de validade discrimante.

\section{DISCUSSÃO}

O objetivo do presente estudo passou por avaliar as propriedades psicométricas da
PHLMS (Cardaciotto et al., 2008), adaptada para a população portuguesa, considerando fumadores e não fumadores. Com isto, sugere-se um instrumento passível de medir duas das principais dimensões do mindfulness (Bishop et al., 2004; Cardaciotto et al., 2008; Silveira et al., 2012), com validação para a língua portuguesa de Portugal e aplicável a amostras sem experiência no treino de meditação baseada em mindfulness (Cardaciotto et al., 2008). 
Cardaciotto et al. (2008) começaram por apresentar bons índices de confiabilidade nas duas subscalas da PHLMS, mantendo-se a evidência em trabalhos seguintes (Teixeira et al., 2017; Tejedor et al., 2014; Silveira et al., 2012). Para a literatura (Marôco, 2021; Pais-Ribeiro, 2010) valores de alfa de Cronbach entre .70 e .89 sugerem níveis qualitativos de confiabilidade entre o satisfatório $(\alpha=.70-$ $\alpha .79)$ e o bom $(\alpha .80-\alpha .89)$. Considerando os valores sugeridos nas investigações prévias à PHLMS e as considerações teóricas sobre os índices de confiabilidade, o nosso estudo mantém bons níveis de consistência interna da escala na mensuração das suas dimensões (consciência e aceitação).

Vários estudos têm alertado para a importância da AFC como método indicado para a validação dos instrumentos em processo de adaptação e de validação (Fernandes \& Vasconcelos-Raposo, 2010; Marôco, 2021; Vasconcelos-Raposo et al., 2012; Vasconcelos-Raposo et al., 2013). Acrescentámos conteúdo sobre a validade da PHLMS, no contexto português, já que os estudos prévios fizeram-no com recurso a uma análise fatorial exploratória (Teixeira et al., 2017). Para Fernandes e Vasconcelos-Raposo (2010) este procedimento pode apresentar uma série de limitações que sujeita as interpretações a uma margem de erro alargada e embora seja um procedimento útil no processo, deve ser procedido da análise fatorial confirmatória (Marôco, 2021). Atendendo à proposta de Cardaciotto et al. (2008), podemos assumir a manutenção do modelo teórico proposto com bons índices de ajustamento (Marôco, 2021), sugerindo assim um modelo bifatorial oblíquo da PHLMS (Cardaciotto et al., 2008; Silveira et al., 2012; Teixeira et al., 2017; Tejedor et al., 2014). Ao contrário da proposta de Zeng et al. (2015), a tradução da PHLMS deste estudo apresentou pesos fatoriais satisfatórios em todos os itens da escala, pelo que se preservou o modelo proposto pelos estudos iniciais (Cardaciotto et al., 2008).
Entre fumadores e não fumadores, a análise da invariância e as evidências permitem aceitar a validade do instrumento quando aplicado a estes diferentes subgrupos (Marôco, 2021; Vasconcelos-Raposo et al., 2013). Conforme reportaram Fernandes e Vasconcelos-Raposo (2010) não proceder à análise de invariância potencia a que a generalização e aplicabilidade do instrumento fiquem comprometidas. Com os resultados obtidos, podemos referir que a versão portuguesa da PHLMS apresenta aptidões na medição das dimensões consciência e aceitação sem distinções entre consumidores e não consumidores de cigarros. Dado o interesse crescente, vários estudos aplicaram intervenções baseadas em mindfulness como estratégia facilitadora do processo de cessação tabágica (Brewer et al., 2013; Elwafi et al., 2012; Luberto et al., 2014), embora no contexto português, as publicações sobre o estudo do impacto dos processos metacognitivos do mindfulness ao nível da cessação tabágica, sejam exíguos.

Algumas limitações deste estudo devem ser identificadas e discutidas. Assim, começamos por referir o caráter transversal da investigação. Este facto não permite conferir a estabilidade dos resultados, passíveis de serem identificados num estudo do tipo longitudinal, por exemplo teste-reteste (Cardaciotto et al., 2008). Porém, importa salientar que a AFC constitui-se como um procedimento de validação robusto e sustentado (Fernandes \& Vasconcelos-Raposo, 2010; Marôco, 2021), permitindo estabelecer um prognóstico favorável quanto ao uso da escala, dados os resultados recolhidos. Outra limitação prende-se com o tipo de amostragem (de conveniência e não probabilística), realizada com uma amostra universitária. Como veiculado na literatura (Marôco, 2021; Pais-Ribeiro, 2010) é limitado proceder a generalizações quando se recorre a este tipo de estratégias de amostragem, embora se tenha procurado controlar o tamanho da amostra, de modo a que esse potencial viés ficasse minimizado. Outro aspeto vai para a discrepância 
entre grupos de pessoas fumadoras e não fumadoras. Este fator pode explicar o valor reduzido quanto ao GFI na análise do modelo fatorial. Contudo, os procedimentos de análise de invariância os indicadores permitem sugerir com segurança a homogeneidade do comportamento da escala entre grupos. Por último, no grupo de fumadores poucos reuniram critérios de classificação para dependência elevada à nicotina (Fargerström, 1978). Porém, face ao consumo de tabaco, as consequências no médio-longo prazo são reais (Fagerström, 1978; Brewer et al., 2013) pelo que o consumo diário de um a quatro cigarros já é tido como um comportamento clinicamente significativo (Elwafi et al., 2012; Fargerström, 1978; Luberto et al., 2014).

Pesquisas futuras podem aprofundar determinadas questões decorrentes da presente investigação. Para além de auxiliar o desenvolvimento de estudos que procurem compreender o impacto dos processos de mindfulness propostos no modelo de Cardaciotto et al. (2008), ao nível da saúde ou do desenvolvimento humano, os estudos de validação poderão ser aprofundados com amostras clínicas e com outras especificidades.

\section{CONCLUSÃO}

Esta pesquisa permitiu acrescentar algumas evidências importantes. Em primeiro lugar, a versão portuguesa da PHLMS apresentou bons níveis de confiabilidade para as duas dimensões principais do mindfulness como verificado em estudos anteriores (Cardaciotto et al., 2008; Silveira et al., 2012; Teixeira et al., 2017). Neste seguimento, a composição fatorial proposta inicialmente (Cardaciotto et al., 2008) foi mantida, com bons índices de ajustamento. Por fim, a análise multigrupos apresentou índices de invariância do modelo bifatorial, na PHLMS para pessoas fumadoras e não fumadoras. Dadas as evidências encontradas, promove-se a produção de novas investigações que atendam a outras componentes metodológicas e com recurso a amostras mais heterogéneas, para assim aprimorar o conhecimento em torno das propriedades psicométricas do instrumento.

\section{Agradecimentos:}

Nada declarado.

\section{Conflito de Interesses:}

Nada declarado.

\section{Financiamento:}

Nada declarado.

\section{REFERÊNCIAS}

Aroian, K., \& Norris, A. (2005). Confirmatory factor analysis. In B. Munro (Ed.), Statistical methods for health care research (5th ed., pp. 351-375). Lippincott Williams \& Wilkins.

Baer, R., Smith, G., Lykins, E., Button, D., Krietemeyer, J., Sauer, S., \& Walsh, E. (2008). Construct validity of the fivefacet mindfulness questionnaire in meditating and nonmeditating samples. Assessment, 15(3), 329-342. https://doi. org/10.1177/1073191107313003

Bishop, S. R., Lau, M., Shapiro, S., Carlson, L., Anderson, N. D., Carmody, J., Segal, Z. V., Abbey, S., Speca, M., Velting, D., \& Devins, G. (2004). Mindfulness: A proposed operational definition. Clinical Psychology: Science and Practice, 11(3), 230-241. https:// doi.org/10.1093/clipsy.bph077

Bloise, P. V., Andrade, M. C., Machado, H., \& Andreoli, S. B. (2016). Increasing awareness and acceptance through mindfulness and somatic education movements. Advances in Mind-body Medicine, 30(4), 4-7. https:// europepmc.org/article/med/27925606

Brewer, J. A., Elwafi, H. M., \& Davis, J. H. (2013). Craving to quit: Psychological models and neurobiological mechanisms of mindfulness training as treatment for addictions. Psychology of Addictive Behaviors, 27(2), 366-379. https://doi.org/10.103/ a0028490. 
Brown, K. W., \& Ryan, R. M. (2003). The benefits of being present: mindfulness and its role in psychological well-being. Journal of Personality and Social Psychology, 84(4), 822. https://doi. org/10.1037/0022-3514.84.4.822.

Browne, M. W., \& Cudeck, R. (1989). Single sample cross-validation indices for covariance structures. Multivariate Behavioral Research, 24(4), 445-455. https://doi. org/10.1207/s15327906mbr2404_4

Buffart, L. M., Singh, A. S., Van Loon, E. C., Vermeulen, H. I., Brug, J., \& Chinapaw, M. J. (2014). Physical activity and the risk of developing lung cancer among smokers: A meta-analysis. Journal of Science and Medicine in Sport, 17(1), 67-71. https://doi. org/10.1016/j.jsams.2013.02.015.

Butryn, M. L., Juarascio, A., Shaw, J., Kerrigan, S. G., Clark, V., O'Planick, A., \& Forman, E. M. (2013). Mindfulness and its relationship with eating disorders symptomatology in women receiving residential treatment. Eating Behaviors, 14(1), 13-16. https://doi. org/10.1016/j.eatbeh.2012.10.005.

Byrne, B. M. (2001). Structural equation modeling with AMOS: Basic concepts, applications and programming. Erlbaum.

Cardaciotto, L., Herbert, J. D., Forman, E. M., Moitra, E., \& Farrow, V. (2008). The assessment of present-moment awareness and acceptance: the Philadelphia Mindfulness Scale. Assessment, 15(2), 204-223. https:// doi.org/10.1177/1073191107311467

Elwafi, H. M., Witkiewitz, K., Mallik, S., Thornhill, T. A., \& Brewer, J. A. (2013). Mindfulness training for smoking cessation: moderation of the relationship between craving and cigarette use. Drug and Alcohol Dependence, 130(1-3), 222-229. https://doi. org/10.1016/j.drugalcdep.2012.11.015

Fagerström, K. O. (1978). Measuring degree of physical dependence to tobacco smoking with reference to individualization of treatment. Addictive Behaviors, 3(3-4), 235-241. https://doi.org/10.1016/0306-
4603(78)90024-2

Fernandes, H. M., \& Vasconcelos-Raposo, J. (2010). Análise factorial confirmatória do TEOSQp. Psicologia: Reflexão e Crítica, 23(1), 92-101. https://doi.org/10.1590/S010279722010000100012.

Grossman, P., \& Van Dam, N. T. (2011). Mindfulness, by any other name...: trials and tribulations of sati in western psychology and science. Contemporary Buddhism, 12(1), 219-239. https://doi.org/10.1080/1463994 7.2011.564841.

Kabat-Zinn, J. (2003). Mindfulness-based interventions in context: Past, present, and future. Clinical Psychology: Science \& Practice, 10(2), 144-156. https://doi.org/10.1093/ clipsy/bpg016.

Lau, M. A., Bishop, S. R., Segal, Z. V., Buis, T., Anderson, N. D., Carlson, L., Shapiro, S., Carmody, J., Abbey, S., \& Devins, G. (2006). The Toronto Mindfulness Scale: development and validation. Journal of Clinical Psychology, 62(12), 1445-1467. https://doi. org/10.1002/jclp.20326

Luberto, C., McLeish, A., Robertson, S., Avallone, K., Kraemer, K., \& Jeffries, E. R. (2014). The role of mindfulness skills in terms of distress tolerance: A pilot test among adult daily smokers. The American Journal on Addictions, 23(2), 184-188. https://doi.org/10.1111/ j.1521-0391.2013.12096.x.

Marôco, J. (2021). Análise de equações estruturais: Fundamentos teóricos, software e aplicações ( $3^{\text {a }}$ ed.). ReportNumber.

Morgan, M. C., Cardaciotto, L., Moon, S., \& Marks, D. (2020). Validation of the Philadelphia Mindfulness Scale on experienced meditators and nonmeditators. Journal of Clinical Psychology, 76(4), 725-748. https://doi.org/10.1002/jclp.22907.

Pais-Ribeiro, J. (2010). Metodologia de investigação em psicologia da saúde ( $3^{\mathrm{a}} \mathrm{ed}$.). Legis Editora.

Rocha, V., Guerra, M. P., \& Maciel, M. J. (2010). Dependência tabágica, assertividade e alexitimia em doentes cardíacos. Paidéia, 
20(46), 155-164. https://doi.org/10.1590/ S0103-863X2010000200003

Rosch, E. (2007). More than mindfulness: when you have a tiger by the tail, let it eat you. Psychological Inquiry, 18(4), 258-264. https:// doi.org/10.1080/10478400701598371.

Segal, Z. V., Williams, J. M. G., \& Teasdale, J. D. (2012). Mindfulness-based cognitive therapy for depression (2nd ed.). Guilford Press.

Silveira, A. D., Castro, T. G., \& Gomes, W. B. (2012). Adaptação e validação da Escala Filadélfia de Mindfulness para adultos brasileiros. PsicoUSF. Braçança Paulista, SP. 17(2), 215-223. https://doi.org/10.1590/ S1413-82712012000200005.

Teixeira, C. M., Caçador, A., Ferreira, T. M., \& Vasconcelos-Raposo, J. (2020). Confirmatory factor analysis of a Portuguese version of the Pittsburgh Sleep Quality Index. PsychTech $\mathcal{E}$ Health Journal, 1(2), 15-23. https://doi. org/10.26580/PTHJ.art6.2018

Teixeira, R. J., Ferreira, G., \& Pereira., M. G. (2017). Portuguese validation of the Cognitive and Affective Mindfulness Scale-Revised and the Philadelphia Mindfulness Scale. Mindfulness \& Compassion, 1(2), 3-8. https://doi.org/10.1016/j. mincom.2017.03.001.

Tejedor, R., Feliu-Soler, A., Pascual, J.C., Cebolla, A., Portella, M., Trujols, J., Soriano, J., Pérez, V., \& Soler, J. (2014). Propiedades psicométricas de la versión española de la Philadelphia Mindfulness Scale. Revista de Psiquiatría y Salud Mental, 7(4), 157-165. https://doi.org/10.1016/j. rpsm.2014.04.001.

Tronieri, J. S., Wadden, T. A., Pearl, R. L., Berkowitz, R. I., Alamuddin, N., \& Chao, A. M. (2020). Mindful Eating, General Mindful Awareness, and Acceptance as Predictors of Weight Loss. Mindfulness, 11(12), 2818-
2827. https://doi.org/10. 1007/s12671-020014935.

Vasconcelos-Raposo, J., Fernandes, H. M., \& Teixeira, C. M. (2013). Factor structure and reliability of the depression, anxiety and stress scales in a large Portuguese community sample. The Spanish Journal of Psychology, 16, 1-10. https://doi.org/10.1017/sjp.2013.15.

Vasconcelos-Raposo, J., Fernandes, H. M., Teixeira, C. M., \& Bertelli, R. (2012). Factorial validity and invariance of the Rosenberg Self-Esteem Scale among Portuguese youngsters. Social Indicators Research, 105(3), 483-498. https://doi. org/10.1007/s11205-011-9782-0.

Walach, H., Buchheld, N., Buttenmüller, V., Kleinknecht, N., \& Schmidt, S. (2006). Measuring mindfulness - the Freiburg mindfulness inventory (FMI). Personality and Individual Differences, 40(8), 1543-1555. https://doi.org/10.1016/j.paid.2005.11.025.

Williams, J. \& Kabat-Zinn, J. (2011). Mindfulness: diverse perspectives on its meaning, origins, and multiple applications at the intersection of science and dharma. Contemporary Buddhism, 12(1), 1-18. https:// doi.org/10.1080/14639947.2011.564811.

Williams, J. \& Penman, D. (2011). Mindfulness: A practical guide to finding peace in a frantic world. Piatkus.

Zeng, X., Li, M., Zhang, B., \& Liu, X. (2015). Revision of the Philadelphia Mindfulness Scale for measuring awareness and equanimity in Goenka's Vipassana meditation with Chinese Buddhists. Journal of religion and health, 54(2), 623-637. https:// doi.org/10.1007/s10943-014-9870-y. 